\title{
アクチュエータ系ダイナミクスを考慮した ロボットの軌道追従フィードバック階層制御
}

全 珍 雨*1 川村 貞 夫*1 金 岡 克 弥*1 櫟弘明*2

\section{A Cascaded Feedback Control Scheme for Trajectory Tracking of Robot Manipulator Systems WITH Actuator Dynamics}

\author{
Jinwoo Jun*1, Sadao Kawamura*1, Katsuya Kanaoka*1 and Hiroaki Ichii*2
}

\begin{abstract}
In the case of control for nonlinear mechanical systems, which have complex actuator dynamics, the total system becomes high-order and nonlinear. Generally speaking, it is not easy to tune feedback gains for state feedback. In this paper, we propose a feedback control scheme for motion control of nonlinear high-order systems. We prove that the proposed scheme can improve the trajectory tracking performance of a robot manipulator system with actuator dynamics. Moreover, some simulation results demonstrate the effectiveness of the proposed control scheme.
\end{abstract}

Key Words: Actuator Dynamics, Nonlinear, High-order Systems, Cascaded Feedback, Real-time Trajectory Tracking

1.は じめに

近年, 各種のアクチュエータが開発され, それらの特徴を考 慮して機械システム制御へ応用される。 その際, 各種のアクチュ エータでは，必ずしも駆動系のダイナミクスが無視できない場 合がある、空気圧駆動システム [1], 形状記憶合金アクチュエー タシステム $[2]$, スラスタ駆動水中ロボット [3], 柔軟関節を持つ ロボットマニピュレータ [4] などがその例である.これらの共通 点は, アクチュエータ系の出力が, 機械システム系への入力と なっていることである。結果的に全体のシステムが高次系にな り，フィードバック制御において，ゲインの設定によっては不 安定となることはよく知られている.さらに, 多くの場合, ア クチュエータ系のダイナミクスは非線形特性を有しており, 通 常の線形システム制御では対応し難い場合も多い.

上記と同じ観点から，一般のロボットなどの機械システムに 時間関数などで設定された目標軌道を高精度に追従させる問題 においてフィードフォワード入力だけに頼らず, フィードバック 入力で可能な限り不安定とならないように追従させるというこ とは, ダイナミクスのパラメータが未知の場合, 実用的メリッ トが大きい。

そこで本論文では，このようなアクチュエータ系ダイナミク

原稿受付 2006 年 7 月 29 日

*1立命館大学理工学部ロボティクス学科

*2 奈良了.業高等専門学校

${ }^{* 1}$ Department of Robotics, the faculty of science and technology, Ritsumeikan University

${ }^{* 2}$ Nara National College of Technology
スを考慮したロボットの制御で, 軌道追従をフィードバック制 御で実現し, 安定性を保証しながら, フィードバックゲインの増 加とともに軌道追従誤差を小さくできる方式を提案する.ただ し，七ンサや信号処理の信頼性の問題点から，ロボットの制御 の状態量としては, 位置と速度信号のみを利用する。ロボット の軌道追従制御は, 線形 PD フィードバックによって, フィー ドバックゲインを増加させることによって, 軌道追従精度が向 上することがいくつかの論文によって示されている [6] [7]. そ こで，ロボットシステムには，この制御入力を構成し，ロボッ トへ与えるフィードバック入力値量をアクチュエータの目標值 とする。次に，このようにして作られたアクチュエータの目標 值に対して, フィードバック入力を構成して, アクチュエータ への入力とする.すなわち, 階層的にフィードバック入力を連 結させた構造となっており，アクチュエータ系のフィードバッ クが, 理想的に目標值を実現すれば，ロボットへの PD フィー ドバック制御を達成していることになる。

このような前段のダイナミクスの目標值を後段のダイナミク スの状態フィードバックで与える手法は, 空気圧駆動システムの 位置決め制御 [1], 柔軟関節を有するロボットマニピュレータ [4], 水中ロボットの軌道制御 [3] などで報告されている，ただし，空 気圧駆動では位置決めの安定性のみを議論している。また, 柔 軟関節ロボットと水中ロボットでは, 適応制御によってフィー ドフォワード入力を形成することに主目的があり，フィードバッ ク制御のゲインの調整などに関しては触れられていない.

本論文では，アクチュエータ系ダイナミクスが1次遅れ系の 非線形システムで表現されるモデルを考える．空気の圧縮性ダ 
イナミクス [1], 形状記憶合金ダイナミクス [2] などは非線形性 を有し，1 次遅れ系で近似される場合が多いからである。そこ で, 1 階の非線形微分方程式でアクチュエータダイナミクスが 表現され，ロボットダイナミクスの入力トルクが発生する場合 を議論する，2 章では，アクチュエータダイナミクスとロボッ トマニピュレータダイナミクスについて説明し，階層的フィー ドバック制御を述べる。次に 3 章に扔いて, 軌道追従性の証明 を行う. 4 章では, 目標值が時間的に変化しない一定值となっ た場合の, 安定性やその他の性質について論じる。最後に 5 章 では, 空気圧駆動システムの場合, 空気圧のダイナミクスに対 して，本論文で提案する手法が適用可能であることを示す。ま た, いくつかの代表的なシミュレーション結果を示し, 提案手 法の有効性を示す。

\section{2. 対象ダイナミクス}

ロボットダイナミクスを次式に与える.

$$
\boldsymbol{R}(\boldsymbol{q}) \ddot{\boldsymbol{q}}+\left[\boldsymbol{B}_{0}+\frac{1}{2} \dot{\boldsymbol{R}}(\boldsymbol{q})+\boldsymbol{S}(\boldsymbol{q}, \dot{\boldsymbol{q}})\right] \dot{\boldsymbol{q}}+\boldsymbol{g}(\boldsymbol{q})=\boldsymbol{\tau}(1)
$$

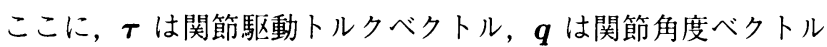
$(n \times 1), \boldsymbol{R}(\boldsymbol{q})$ は慣性行列, $\boldsymbol{B}_{0}$ はロボット関節の粘性行列, $\boldsymbol{S}(\boldsymbol{q}, \dot{\boldsymbol{q}})$ は遠心・コリオリカを表す。

このロボットダイナミクスに対して, ロボットの目標軌道, $\boldsymbol{q}_{d}(t)$ が時間， $t \geq 0$ において以下のように与えられるとする.

(1) $\boldsymbol{q}_{d}(t) \in \mathrm{C}^{2}\left(\boldsymbol{q}_{d}(t)\right.$ の 2 階微分が連続 $)$,

(2) $q_{d i}(0)=\dot{q}_{d i}(0)=0$

(3) $\ddot{q}_{d i}(0)=\dot{q}_{d i}(0)=0, \quad t \geq T_{f}$.

ただし， $q_{d i}(i=1,2, \ldots, n)$ は $\boldsymbol{q}$ の各要素である.すなわち, $0 \leq t \leq T_{f}$ において運動が発生し, $t \geq T_{f}$ において一定值と なる，次にアクチュエータダイナミクスを次式のようにモデル 化する。

$$
\dot{\boldsymbol{\tau}}+\boldsymbol{A}(\boldsymbol{q}, \dot{\boldsymbol{q}}) \boldsymbol{\tau}=\boldsymbol{D}(\boldsymbol{q}, \dot{\boldsymbol{q}}, \boldsymbol{\tau}) \boldsymbol{u}
$$

とする. ただし， $\boldsymbol{u}$ はアクチュエータへの入力ベクトル $(n \times 1)$, $\boldsymbol{A}(\boldsymbol{q}, \dot{\boldsymbol{q}}), \boldsymbol{D}(\boldsymbol{q}, \dot{\boldsymbol{q}}, \boldsymbol{\tau})$ は非線形の係数行列であり, 以下の条件 を満足するものとする.

[条件 1] 任意の $\boldsymbol{q}_{1}, \boldsymbol{q}_{2}$ に対して, 適当な大きさの定数 $c_{1}$, $c_{2}$ が存在し, 任意の時刻 $(t \geq 0)$ において以下の不等式を満足 する.

$$
\begin{aligned}
& \left\|\boldsymbol{A}\left(\boldsymbol{q}_{1}, \dot{\boldsymbol{q}}_{1}\right)-\boldsymbol{A}\left(\boldsymbol{q}_{2}, \dot{\boldsymbol{q}}_{2}\right)\right\| \\
& \quad \leq c_{1}\left\|\boldsymbol{q}_{1}(t)-\boldsymbol{q}_{2}(t)\right\|+c_{2}\left\|\dot{\boldsymbol{q}}_{1}(t)-\dot{\boldsymbol{q}}_{2}(t)\right\|
\end{aligned}
$$

[条件 2] 目標軌道 $\boldsymbol{q}_{d}(t)$ に対して, 適当な正数 $c_{3}$ によって

$$
\left\|\boldsymbol{A}\left(\boldsymbol{q}_{d}, \dot{\boldsymbol{q}}_{d}\right)\right\| \leq c_{3}
$$

[条件 3] 適当な関数行列 $\boldsymbol{H}(\boldsymbol{q}, \dot{\boldsymbol{q}}, \boldsymbol{\tau})$ によって, 任意の $\boldsymbol{q}$, $\dot{\boldsymbol{q}}, \boldsymbol{\tau}$ に対して, 適当な大きさの正数 $c_{4}, c_{5}$ が存在し, 以下を 満足する。

$$
\mathbf{0}<c_{4} \boldsymbol{I} \leq \boldsymbol{D}(\boldsymbol{q}, \dot{\boldsymbol{q}}, \boldsymbol{\tau}) \boldsymbol{H}(\boldsymbol{q}, \dot{\boldsymbol{q}}, \boldsymbol{\tau}) \leq c_{5} \boldsymbol{I}
$$

ただし，Iは単位行列である。

ここでロボットへの理想的なフィードバック入力 $\boldsymbol{\tau}_{d}$ を次式 で与える。

$$
\boldsymbol{\tau}_{d}=-\boldsymbol{G}_{p} \Delta \boldsymbol{q}-\boldsymbol{G}_{v} \Delta \dot{\boldsymbol{q}}+\boldsymbol{g}\left(\boldsymbol{q}_{d}\right)
$$

ここに, $\Delta \boldsymbol{q}=\boldsymbol{q}-\boldsymbol{q}_{d}(n \times 1), \boldsymbol{G}_{p}$ : 位置フィードバックゲイ ン行列 $(n \times n), \boldsymbol{G}_{v}$ : 速度フィードバックゲイン行列 $(n \times n)$ で, 右辺第 3 項は重力補償項である.

フィードバックゲインについては,

$$
\boldsymbol{G}_{p}=a \rho \boldsymbol{I}, \boldsymbol{G}_{v}=\rho \boldsymbol{I}
$$

とする.ここに $a>0$ はフィードバックゲインの比率を決める パラメータであり，以下の議論ではゲインの大きさは $\rho>0$ に よって調節されるとする。 これは, 以下で安定なゲイン調整の 条件導出を容易に行うためである.

アクチュエータ系への入力を以下で与える.

$$
\begin{aligned}
\boldsymbol{u}= & -\boldsymbol{H}(\boldsymbol{q}, \dot{\boldsymbol{q}}, \boldsymbol{\tau}) \boldsymbol{K}_{p} \Delta \boldsymbol{\tau} \\
& +\boldsymbol{H}(\boldsymbol{q}, \dot{\boldsymbol{q}}, \boldsymbol{\tau}) \hat{\boldsymbol{A}}\left(\boldsymbol{q}_{d}, \dot{\boldsymbol{q}}_{d}\right) \boldsymbol{g}\left(\boldsymbol{q}_{d}\right)
\end{aligned}
$$

ここで, $\Delta \boldsymbol{\tau}=\boldsymbol{\tau}-\boldsymbol{\tau}_{d}$, 行列 $\boldsymbol{H}(\boldsymbol{q}, \dot{\boldsymbol{q}}, \boldsymbol{\tau})$ は, 式（5）の条件を 満足する行列を利用する. また $\hat{\boldsymbol{A}}(\boldsymbol{q}, \dot{\boldsymbol{q}})$ は, $\boldsymbol{A}(\boldsymbol{q}, \dot{\boldsymbol{q}})$ の推定行 列である。ここでは, 任意の $\boldsymbol{q}, \dot{\boldsymbol{q}}$ に対して $\hat{\boldsymbol{A}}(\boldsymbol{q}, \dot{\boldsymbol{q}})$ は有界と する. 次に, この理想入力 $\boldsymbol{\tau}_{d}$ を式（1）に代入して次式を得る.

$$
\begin{gathered}
\boldsymbol{R}(\boldsymbol{q}) \Delta \ddot{\boldsymbol{q}}+\left[\boldsymbol{B}_{0}+\frac{1}{2} \dot{\boldsymbol{R}}(\boldsymbol{q})+\boldsymbol{S}(\boldsymbol{q}, \dot{\boldsymbol{q}})\right] \Delta \dot{\boldsymbol{q}}+\boldsymbol{G}_{v} \Delta \dot{\boldsymbol{q}} \\
+\boldsymbol{G}_{p} \Delta \boldsymbol{q}+\boldsymbol{h}\left(\Delta \boldsymbol{q}, \Delta \dot{\boldsymbol{q}}, \boldsymbol{q}_{d}, \dot{\boldsymbol{q}}_{d}, \ddot{\boldsymbol{q}}_{d}\right)=\Delta \boldsymbol{\tau}+\boldsymbol{\psi}_{q}
\end{gathered}
$$

$$
\begin{aligned}
\boldsymbol{h} & \left(\Delta \boldsymbol{q}, \Delta \dot{\boldsymbol{q}}, \boldsymbol{q}_{d}, \dot{\boldsymbol{q}}_{d}, \ddot{\boldsymbol{q}}_{d}\right)=\left\{\boldsymbol{R}(\boldsymbol{q})-\boldsymbol{R}\left(\boldsymbol{q}_{d}\right)\right\} \ddot{\boldsymbol{q}}_{d} \\
& +\left\{\frac{1}{2} \dot{\boldsymbol{R}}(\boldsymbol{q})+\boldsymbol{S}(\boldsymbol{q}, \dot{\boldsymbol{q}})-\frac{1}{2} \dot{\boldsymbol{R}}\left(\boldsymbol{q}_{d}\right)-\boldsymbol{S}\left(\boldsymbol{q}_{d}, \boldsymbol{q}_{d}\right)\right\} \dot{\boldsymbol{q}}_{d} \\
& +\boldsymbol{g}(\boldsymbol{q})-\boldsymbol{g}\left(\boldsymbol{q}_{d}\right)
\end{aligned}
$$

ただし，

$$
\boldsymbol{\psi}_{q}=-\left[\boldsymbol{R}\left(\boldsymbol{q}_{d}\right) \ddot{\boldsymbol{q}}_{d}+\left\{\boldsymbol{B}_{0}+\frac{1}{2} \dot{\boldsymbol{R}}\left(\boldsymbol{q}_{d}\right)+\boldsymbol{S}\left(\boldsymbol{q}_{d}, \dot{\boldsymbol{q}}_{d}\right)\right\} \dot{\boldsymbol{q}}_{d}\right]
$$

であり, 文献 [5] に示されるように, $\boldsymbol{h}\left(\Delta \boldsymbol{q}, \Delta \dot{\boldsymbol{q}}, \boldsymbol{q}_{d}, \dot{\boldsymbol{q}}_{d}, \ddot{\boldsymbol{q}}_{d}\right)$ は, その構造から適当な正数 $c_{6}, c_{7}$ によって以下を満足する.

$$
\left\|\boldsymbol{h}\left(\Delta \boldsymbol{q}, \Delta \dot{\boldsymbol{q}}, \boldsymbol{q}_{d}, \dot{\boldsymbol{q}}_{d}, \ddot{\boldsymbol{q}}_{d}\right)\right\| \leq c_{6}\|\Delta \boldsymbol{q}\|+c_{7}\|\Delta \dot{\boldsymbol{q}}\|
$$

ここで, $\dot{\boldsymbol{q}}_{d}=\ddot{\boldsymbol{q}}_{d}=\mathbf{0}$ のとき, $\boldsymbol{\psi}_{q}=\mathbf{0}$ となることに注意する.

次に, アクチュエータのダイナミクスについて式 (2)，(6)，

（8）により，次式になる.

$$
\begin{aligned}
\Delta \dot{\boldsymbol{\tau}} & +\boldsymbol{A}(\boldsymbol{q}, \dot{\boldsymbol{q}}) \Delta \boldsymbol{\tau} \\
= & \boldsymbol{D}(\boldsymbol{q}, \dot{\boldsymbol{q}}, \boldsymbol{\tau})\left\{-\boldsymbol{H}(\boldsymbol{q}, \dot{\boldsymbol{q}}, \boldsymbol{\tau}) \boldsymbol{K}_{p} \Delta \boldsymbol{\tau}\right. \\
& \left.+\boldsymbol{H}(\boldsymbol{q}, \dot{\boldsymbol{q}}, \boldsymbol{\tau}) \hat{\boldsymbol{A}}\left(\boldsymbol{q}_{d}, \dot{\boldsymbol{q}}_{d}\right) \boldsymbol{g}\left(\boldsymbol{g}_{d}\right)\right\} \\
& -\dot{\boldsymbol{\tau}}_{d}-\boldsymbol{A}(\boldsymbol{q}, \dot{\boldsymbol{q}}) \boldsymbol{\tau}_{d}
\end{aligned}
$$

上式に式（6）の $\boldsymbol{\tau}_{d}$ を代入する際， $\Delta \ddot{\boldsymbol{q}}$ の項が $\dot{\boldsymbol{\tau}}_{d}$ に含まれる ので，式（9）を代入する．結果として以下となる. 


$$
\begin{aligned}
\boldsymbol{G}_{v}^{-1} & \Delta \dot{\boldsymbol{\tau}}+\left\{\boldsymbol{G}_{v}^{-1} \boldsymbol{A}(\boldsymbol{q}, \dot{\boldsymbol{q}})\right. \\
& \left.+\boldsymbol{G}_{v}^{-1} \boldsymbol{D}(\boldsymbol{q}, \dot{\boldsymbol{q}}, \boldsymbol{\tau}) \boldsymbol{H}(\boldsymbol{q}, \dot{\boldsymbol{q}}, \boldsymbol{\tau}) \boldsymbol{K}_{p}-\boldsymbol{R}^{-1}(\boldsymbol{q})\right\} \Delta \boldsymbol{\tau} \\
& +\left[\boldsymbol{R}^{-1}(\boldsymbol{q})\left\{\boldsymbol{B}_{0}+\frac{1}{2} \dot{\boldsymbol{R}}(\boldsymbol{q})+\boldsymbol{S}(\boldsymbol{q}, \dot{\boldsymbol{q}})+\boldsymbol{G}_{v}\right\}\right. \\
& \left.-\boldsymbol{G}_{v}^{-1} \boldsymbol{G}_{p}-\boldsymbol{G}_{v}^{-1} \boldsymbol{A}(\boldsymbol{q}, \dot{\boldsymbol{q}}) \boldsymbol{G}_{v}\right] \Delta \dot{\boldsymbol{q}} \\
& +\left[\boldsymbol{R}^{-1}(\boldsymbol{q}) \boldsymbol{G}_{p}-\boldsymbol{G}_{v}^{-1} \boldsymbol{A}(\boldsymbol{q}, \dot{\boldsymbol{q}}) \boldsymbol{G}_{p}\right] \Delta \boldsymbol{q} \\
& +\boldsymbol{R}^{-1}(\boldsymbol{q}) \boldsymbol{h}\left(\Delta \boldsymbol{q}, \Delta \dot{\boldsymbol{q}}, \boldsymbol{q}_{d}, \dot{\boldsymbol{q}}_{d}, \ddot{\boldsymbol{q}}_{d}\right) \\
& +\boldsymbol{G}_{v}^{-1}\left\{\boldsymbol{A}(\boldsymbol{q}, \dot{\boldsymbol{q}})-\boldsymbol{A}\left(\boldsymbol{q}_{d}, \dot{\boldsymbol{q}}_{d}\right)\right\} \boldsymbol{g}\left(\boldsymbol{q}_{d}\right)=\boldsymbol{\psi}_{a}
\end{aligned}
$$

ただし，

$$
\begin{aligned}
\boldsymbol{\psi}_{a}= & \boldsymbol{R}^{-1}(\boldsymbol{q}) \boldsymbol{\psi}_{q}+\boldsymbol{G}_{v}^{-1}\left[-\dot{\boldsymbol{g}}\left(\boldsymbol{q}_{d}\right)\right. \\
& +\left\{\boldsymbol{D}(\boldsymbol{q}, \dot{\boldsymbol{q}}, \boldsymbol{\tau}) \boldsymbol{H}(\boldsymbol{q}, \dot{\boldsymbol{q}}, \boldsymbol{\tau}) \hat{\boldsymbol{A}}\left(\boldsymbol{q}_{d}, \dot{\boldsymbol{q}}_{d}\right)\right. \\
& \left.\left.-\boldsymbol{A}\left(\boldsymbol{q}_{d}, \dot{\boldsymbol{q}}_{d}\right)\right\} \boldsymbol{g}\left(\boldsymbol{q}_{d}\right)\right]
\end{aligned}
$$

最後にロボットダイナミクスの式 (9) とアクチュエータダイ ナミクスの式（13）において，次の初期条件を設定する.

$$
\begin{gathered}
\Delta \boldsymbol{q}(0)=\boldsymbol{q}(0)-\boldsymbol{q}_{d}(0)=0 ; \Delta \dot{\boldsymbol{q}}(0)=\dot{\boldsymbol{q}}(0)-\dot{\boldsymbol{q}}_{d}(0)=0 \\
\Delta \boldsymbol{\tau}(0)=\boldsymbol{\tau}(0)-\boldsymbol{\tau}_{d}(0)=0
\end{gathered}
$$

\section{3. 軌道追従制御性能}

次にリアプノフ関数, $V(\Delta \boldsymbol{x})$ の候補を考える.

$$
V(\Delta \boldsymbol{x}(t))=\frac{1}{2} \Delta \boldsymbol{x}^{T}(t) \boldsymbol{N} \Delta \boldsymbol{x}(t)
$$

ここに, $\Delta \boldsymbol{x}=\left[\Delta \boldsymbol{q}^{T} \Delta \dot{\boldsymbol{q}}^{T} \Delta \boldsymbol{\tau}^{T}\right]^{T}$ であり, 行列 $\boldsymbol{N}$ は以下で 表現される.

$$
\boldsymbol{N}=\left[\begin{array}{ccc}
\boldsymbol{G}_{p}+\alpha \boldsymbol{G}_{v} & \alpha \boldsymbol{R} & \mathbf{0} \\
\alpha \boldsymbol{R} & \boldsymbol{R} & \mathbf{0} \\
\mathbf{0} & \mathbf{0} & \boldsymbol{G}_{v}^{-1}
\end{array}\right]
$$

ここで慣性行列 $\boldsymbol{R}(\boldsymbol{q})$ は正定対称行列であり，任意の $\boldsymbol{q}$ に対し て, 適当な正数 $c_{8}, c_{9}$ が存在し,

$$
c_{8} \leq\|\boldsymbol{R}(\boldsymbol{q})\| \leq c_{9}
$$

となることはよく知られているので以下の条件を満たせば，行 列 $\boldsymbol{N}$ は正定対称行列となる.

$$
\begin{gathered}
1-\alpha \epsilon>0, \\
\boldsymbol{G}_{p}+\alpha \boldsymbol{G}_{v}-\frac{\alpha}{\epsilon} \boldsymbol{R}>\mathbf{0}
\end{gathered}
$$

ただし， $\alpha, \epsilon$ は適当な正数とする.

ここで $V(\Delta x)$ の時間微分を計算すると次式となる.

$$
\begin{aligned}
\dot{V}(\Delta \boldsymbol{x})= & -\Delta \boldsymbol{x}^{T} \boldsymbol{D} \Delta \boldsymbol{x}+\Delta \boldsymbol{x}^{T} \boldsymbol{\psi} \\
& +\Delta \boldsymbol{x}^{T} \boldsymbol{E} \Delta \boldsymbol{x}+\Delta \boldsymbol{x}^{T} \boldsymbol{\nu}
\end{aligned}
$$

ただし，

$$
\boldsymbol{\psi}=\left[\begin{array}{lll}
\alpha \boldsymbol{\psi}_{q}^{T} & \boldsymbol{\psi}_{q}^{T} & \boldsymbol{\psi}_{a}^{T}
\end{array}\right]^{T},
$$

$$
\boldsymbol{\nu}=\left[\alpha \boldsymbol{h}^{T}-\boldsymbol{h}^{T}-\left(\boldsymbol{R}^{-1}(\boldsymbol{q}) \boldsymbol{h}\right)^{T}\right]^{T}
$$

行列 Dの各ブロック行列要素 $\mathrm{D}_{i j}$ は以下に示される.

$$
\begin{aligned}
\mathrm{D}_{11}= & \alpha \boldsymbol{G}_{p}, \\
\mathrm{D}_{12}= & \mathrm{D}_{21}{ }^{T}=\frac{\alpha}{4}\left(\dot{\boldsymbol{R}}\left(\boldsymbol{q}_{d}\right)+2 \boldsymbol{S}\left(\boldsymbol{q}_{d}, \dot{\boldsymbol{q}}_{d}\right)\right), \\
\mathrm{D}_{22}= & \boldsymbol{G}_{v}+\boldsymbol{B}_{0}-\alpha \boldsymbol{R}, \\
\mathrm{D}_{13}{ }^{T}= & \mathrm{D}_{31}=\frac{1}{2}\left(\alpha \boldsymbol{I}-\boldsymbol{R}^{-1}(\boldsymbol{q}) \boldsymbol{G}_{p}+\boldsymbol{G}_{v}^{-1} \boldsymbol{A}\left(\boldsymbol{q}_{d}, \dot{\boldsymbol{q}}_{d}\right) \boldsymbol{G}_{p}\right), \\
\mathrm{D}_{23}{ }^{T}= & \mathrm{D}_{32}=\frac{1}{2}\left(\boldsymbol{I}-\boldsymbol{B}_{0}-\boldsymbol{G}_{v}+\boldsymbol{G}_{v}^{-1} \boldsymbol{G}_{p}-\frac{1}{2} \dot{\boldsymbol{R}}\left(\boldsymbol{q}_{d}\right)\right. \\
& \left.-\boldsymbol{S}\left(\boldsymbol{q}_{d}, \dot{\boldsymbol{q}}_{d}\right)+\boldsymbol{G}_{v}^{-1} \boldsymbol{A}\left(\boldsymbol{q}_{d}, \dot{\boldsymbol{q}}_{d}\right) \boldsymbol{G}_{v}\right) \\
\mathrm{D}_{33}= & \boldsymbol{G}_{v}^{-1} \boldsymbol{D}(\boldsymbol{q}, \dot{\boldsymbol{q}}, \boldsymbol{\tau}) \boldsymbol{H}(\boldsymbol{q}, \dot{\boldsymbol{q}}, \boldsymbol{\tau}) \boldsymbol{K}_{p}+\boldsymbol{G}_{v}^{-1} \boldsymbol{A}\left(\boldsymbol{q}_{d}, \dot{\boldsymbol{q}}_{d}\right) \\
& -\boldsymbol{R}^{-1}
\end{aligned}
$$

次に, 行列 $\boldsymbol{E}$ の各ブロック行列 $\mathrm{E}_{i j}$ は以下となる.

$$
\begin{aligned}
\mathrm{E}_{11}= & \mathrm{E}_{22}=\mathbf{0}, \\
\mathrm{E}_{12}= & \mathrm{E}_{21}{ }^{T}=\frac{\alpha}{2}\left(\frac{1}{2}\left(\dot{\boldsymbol{R}}(\boldsymbol{q})-\dot{\boldsymbol{R}}\left(\boldsymbol{q}_{d}\right)\right)\right. \\
& +\left(\boldsymbol{S}(\boldsymbol{q}, \dot{\boldsymbol{q}})-\boldsymbol{S}\left(\boldsymbol{q}_{d}, \dot{\boldsymbol{q}}_{d}\right)\right), \\
\mathrm{E}_{13}{ }^{T}= & \mathrm{E}_{31}=\frac{1}{2} \boldsymbol{G}_{v}^{-1}\left(\boldsymbol{A}(\boldsymbol{q}, \dot{\boldsymbol{q}})-\boldsymbol{A}\left(\boldsymbol{q}_{d}, \dot{\boldsymbol{q}}_{d}\right)\right) \boldsymbol{G}_{p}, \\
\mathrm{E}_{23}{ }^{T}= & \mathrm{E}_{32}=-\frac{1}{2}\left(\dot{\boldsymbol{R}}(\boldsymbol{q})-\dot{\boldsymbol{R}}\left(\boldsymbol{q}_{d}\right)\right)+\boldsymbol{S}(\boldsymbol{q}, \dot{\boldsymbol{q}})-\boldsymbol{S}\left(\boldsymbol{q}_{d}, \dot{\boldsymbol{q}}_{d}\right) \\
& -\boldsymbol{G}_{v}^{-1}\left(\boldsymbol{A}(\boldsymbol{q}, \dot{\boldsymbol{q}})-\boldsymbol{A}\left(\boldsymbol{q}_{d}, \dot{\boldsymbol{q}}_{d}\right)\right) \boldsymbol{G} \boldsymbol{G}_{v}, \\
\mathrm{E}_{33}= & -\boldsymbol{G}_{v}^{-1}\left(\boldsymbol{A}(\boldsymbol{q}, \dot{\boldsymbol{q}})-\boldsymbol{A}\left(\boldsymbol{q}_{d}, \dot{\boldsymbol{q}}_{d}\right)\right) \\
\text { 式 }(11) & \text { の } \boldsymbol{h}\left(\Delta \boldsymbol{q}, \Delta \dot{\boldsymbol{q}}, \boldsymbol{q}_{d}, \dot{\boldsymbol{q}}_{d}, \ddot{\boldsymbol{q}}_{d}\right) \text { の性質により以下となる. } \\
& \left\|\alpha \Delta \boldsymbol{q}^{T} h\right\| \leq \alpha c_{10}\|\Delta \boldsymbol{q}\|^{2}+\alpha c_{11}\|\Delta \boldsymbol{q}\|\|\Delta \dot{\boldsymbol{q}}\| \\
& \left\|\Delta \dot{\boldsymbol{q}}^{T} h\right\| \leq c_{12}\|\Delta \boldsymbol{q}\|\|\Delta \dot{\boldsymbol{q}}\|+\alpha c_{13}\|\Delta \dot{\boldsymbol{q}}\|^{2} \\
& \left\|\Delta \boldsymbol{\tau}^{T} \boldsymbol{R}^{-1}(\boldsymbol{q}) h\right\| \leq c_{14}\|\Delta \boldsymbol{q}\|\|\Delta \boldsymbol{\tau}\| \\
& \quad+c_{15}\|\Delta \dot{\boldsymbol{q}}\|\|\Delta \boldsymbol{\tau}\|
\end{aligned}
$$

ただし， $c_{10}, c_{11}, c_{12}, c_{13}, c_{14}, c_{15}$ は適当な正数である. そこで, 式 (21) 加適当な正数 $\epsilon_{1}, \epsilon_{2}, \epsilon_{3}$ によって以下の関 係を得る。

$$
\begin{aligned}
& \left\|\Delta \boldsymbol{x}^{T} \boldsymbol{\nu}\right\| \\
& \quad \leq\left(\alpha c_{9}+\frac{\alpha \epsilon_{1} c_{10}}{2}+\frac{\epsilon_{2} c_{11}}{2}+\frac{\epsilon_{3} c_{13}}{2}\right)\|\Delta \boldsymbol{q}\|^{2} \\
& +\left(c_{12}+\frac{\alpha c_{10}}{2 \epsilon_{1}}+\frac{c_{11}}{2 \epsilon_{2}}+\frac{\epsilon_{3} c_{14}}{2}\right)\|\Delta \dot{\boldsymbol{q}}\|^{2} \\
& +\frac{c_{14}}{2 \epsilon_{3}}\|\Delta \boldsymbol{\tau}\|^{2}
\end{aligned}
$$

よって, 式（19）から以下の式を得る.

$$
\begin{aligned}
\dot{V}(\Delta \boldsymbol{x}) \leq & -\Delta \boldsymbol{x}^{T} \boldsymbol{M} \Delta \boldsymbol{x}+\Delta \boldsymbol{x}^{T} \boldsymbol{\psi} \\
& +\Delta \boldsymbol{x}^{T} \boldsymbol{E} \Delta \boldsymbol{x}
\end{aligned}
$$

ここで, 行列 $\boldsymbol{M}$ の各ブロック行列 $\mathrm{M}_{i j}$ は以下で与えられる. 


$$
\begin{aligned}
& \mathrm{M}_{11}=\mathrm{D}_{11}-\left[\alpha c_{9}+\frac{1}{2}\left(\alpha \epsilon_{1} c_{10}+\epsilon_{2} c_{11}+\epsilon_{3} c_{13}\right)\right] \boldsymbol{I}, \\
& \mathrm{M}_{22}=\mathrm{D}_{22}-\left[c_{12}+\frac{1}{2}\left(\frac{\alpha c_{10}}{\epsilon_{1}}+\frac{c_{11}}{\epsilon_{2}}+\epsilon_{3} c_{14}\right)\right] \boldsymbol{I}, \\
& \mathrm{M}_{33}=\mathrm{D}_{33}-\frac{c_{14}}{2 \epsilon_{3}} \boldsymbol{I}, \quad \mathrm{M}_{i j}=\mathrm{D}_{i j}(i \neq j)
\end{aligned}
$$

次に, 式（19）の右辺第 3 項の行列 $\boldsymbol{E}$ は，各ブロック行列が リプシッツ条件を満足することから適当な正数 $\widetilde{\eta}_{3}$ によって次 式を得る.

$$
\|\boldsymbol{E}\| \leq \widetilde{\eta}_{3}\|\Delta \boldsymbol{x}\|
$$

ここで, 行列 $\boldsymbol{E}$ の要素内にフィードバックゲイン $\boldsymbol{G}_{p}, \boldsymbol{G}_{v}$ を 含む. しかし, $\boldsymbol{G}_{p}$ と $\boldsymbol{G}_{v}$ の $\rho$ を増加させて同様に増加させる ことによって， $\widetilde{\eta}_{3}$ は増加しないことに注意されたい.さらに式 (4)，（5）と $\hat{\boldsymbol{A}}\left(\boldsymbol{q}_{d}, \dot{\boldsymbol{q}}_{d}\right)$ も有界であるので, 次式となる.

$$
\|\boldsymbol{\psi}\| \leq \widetilde{\eta}_{1}
$$

次に, 行列 $\boldsymbol{M}$ において, 行列 $\boldsymbol{M}$ が正定対称行列となる ように $\boldsymbol{G}_{p}, \boldsymbol{G}_{v}$ は十分に大きく設定する. ここで $\boldsymbol{G}_{v}=\rho \boldsymbol{I}$, $\boldsymbol{K}_{p}=\omega \rho^{2} \boldsymbol{I}(\omega>0)$ とすると

$$
\begin{aligned}
\mathrm{D}_{33}= & \omega \rho \boldsymbol{D}(\boldsymbol{q}, \dot{\boldsymbol{q}}, \boldsymbol{\tau}) \boldsymbol{H}(\boldsymbol{q}, \dot{\boldsymbol{q}}, \boldsymbol{\tau}) \\
& -\boldsymbol{R}^{-1}(\boldsymbol{q})+\frac{1}{\rho} \boldsymbol{A}\left(\boldsymbol{q}_{d}, \dot{\boldsymbol{q}}_{d}\right)
\end{aligned}
$$

となる，さらに，十分大きな正数 $\rho$ を設定すれば条件式（18） より $\mathrm{D}_{33}>0$ となり, 適切な大きさの $\omega$ を設定することで行 列 $M$ は正定対称行列となる.

式 $(27) （ 29)$ により以下となる.

$$
\dot{V}(\Delta \boldsymbol{x}) \leq \widetilde{\eta}_{3}\|\Delta \boldsymbol{x}(t)\|^{3}-\widetilde{\eta}_{2}\|\Delta \boldsymbol{x}(t)\|^{2}+\widetilde{\eta}_{1}\|\Delta \boldsymbol{x}(t)\|
$$

ここに,

$$
\tilde{\eta}_{2}=\lambda_{\min } \boldsymbol{M}>0
$$

ただし, $\lambda_{\min } \boldsymbol{Q}$ は行列 $\boldsymbol{Q}$ の最小固有值を表す.

ここで式（30）の右辺について考える. 係数 $\widetilde{\eta}_{1}, \widetilde{\eta}_{3}$ はフィー ドバックゲインの大きさ $\rho$ の増大に伴って, 大きくならない. 一方， $\tilde{\eta}_{2}$ は $\rho$ が大きくなるに従って大きな值として設定でき る.より詳しく調べるために，以下を考える.

パラメー夕 $a, \alpha, \omega$ を適切に設定すれば容易に次の関係が 成立する。

$$
a \alpha \boldsymbol{I}-\frac{a \epsilon_{1}}{2} \boldsymbol{R}^{-1}(\boldsymbol{q})>\frac{1}{2} \boldsymbol{I}
$$

$\omega \boldsymbol{D}(\boldsymbol{q}, \dot{\boldsymbol{q}}, \boldsymbol{\tau}) \boldsymbol{H}(\boldsymbol{q}, \dot{\boldsymbol{q}}, \boldsymbol{\tau})-\frac{a}{2 \epsilon_{1}} \boldsymbol{R}^{-1}(\boldsymbol{q})-\frac{1}{2 \epsilon_{2}} \boldsymbol{I}>\frac{1}{2} \boldsymbol{I}$

ただし， $2>\epsilon_{2}>0$.

よって，十分大きな $\rho$ によって以下の関係が成立することに 注意する.

$$
\boldsymbol{M}>\boldsymbol{M}_{0}>\mathbf{0}
$$

ここで $\boldsymbol{M}_{0}=\frac{\rho}{2} \boldsymbol{I}(3 n \times 3 n)$ である.したがって式（30）に代 わって次式を得る.

$$
\dot{V}(\Delta \boldsymbol{x}) \leq \eta_{3}\|\Delta \boldsymbol{x}(t)\|^{3}-\eta_{2}\|\Delta \boldsymbol{x}(t)\|^{2}+\eta_{1}\|\Delta \boldsymbol{x}(t)\|
$$

ここに $\eta_{1}=\widetilde{\eta}_{1}, \eta_{2}=\lambda_{\min } \boldsymbol{M}_{0}=\rho, \eta_{3}=\widetilde{\eta}_{3}$ である.

ここで, 式 (35) の右辺が偩の部分を持つためには,

$$
\eta_{2}^{2}-4 \eta_{1} \eta_{3}=\frac{\rho^{2}}{4}-4 \eta_{1} \eta_{3}>0
$$

を満足する必要がある。これは適切に大きなフィードバックゲ インの大きさ $\rho$ によって実現できる．したがって，以下の $r_{1}$, $r_{2}$ を根とする 3 次曲線を描く.

$$
r_{1}=\frac{\rho-\sqrt{\rho^{2}-16 \eta_{1} \eta_{3}}}{4 \eta_{3}}, r_{2}=\frac{\rho+\sqrt{\rho^{2}-16 \eta_{1} \eta_{3}}}{4 \eta_{3}}
$$

以下の基本的な考え方は文献 [7] と同様となる.

$\|\Delta \boldsymbol{x}(t)\|>r_{1}$ の場合について議論すればよい. 時刻 $t=t_{0}$ で

$$
\left\|\Delta \boldsymbol{x}\left(t_{0}\right)\right\|=r_{1}
$$

となるとする. 次に, $\delta$ を正の定数として時刻 $t=t_{1}$ で

$$
t=t_{1}=t_{0}+\delta>t_{0}
$$

とする.このとき $\left\|\Delta \boldsymbol{x}\left(t_{1}\right)\right\|>r_{1}$ になったとする．このとき， 次の関係が成立する.

$$
\begin{aligned}
& \lambda_{\min } \boldsymbol{N}\left\|\Delta \boldsymbol{x}\left(t_{1}\right)\right\|^{2} \leq V\left(\Delta \boldsymbol{x}\left(t_{1}\right)\right)<V\left(\Delta \boldsymbol{x}\left(t_{0}\right)\right) \\
& \quad \leq \lambda_{\max } \boldsymbol{N}\left\|\Delta \boldsymbol{x}\left(t_{0}\right)\right\|^{2}=\lambda_{\max } \boldsymbol{N} r_{1}^{2}
\end{aligned}
$$

ただし $\lambda \max \boldsymbol{N}$ : 行列 $\boldsymbol{N}$ の最大固有値とする.

したがって, 次式を得る。

$$
\left\|\Delta \boldsymbol{x}\left(t_{1}\right)\right\| \leq \sqrt{\frac{\lambda_{\max } \boldsymbol{N}}{\lambda_{\min } \boldsymbol{N}}} r_{1}
$$

時刻 $t=t_{0}$ で $\left\|\Delta \boldsymbol{x}\left(t_{0}\right)\right\|=r_{1}$ の大きさを持っていた場合, $t_{1}>t_{0}$ 時刻でも式 (41) の範囲にとどまることが分かる.

以下で, 式（15）の行列 $\boldsymbol{N}$ の各ブロック行列の大きさを論 じる。まず，次の関係に注意する。

$$
\boldsymbol{N}_{1} \leq \boldsymbol{N} \leq \boldsymbol{N}_{2}
$$

ここで

$$
\boldsymbol{N}_{i}=\left[\begin{array}{ccc}
\boldsymbol{G}_{p}+\alpha \boldsymbol{G}_{v} \mp \frac{\alpha}{\epsilon} \boldsymbol{R} & \mathbf{0} & \mathbf{0} \\
\mathbf{0} & (1 \mp \alpha \epsilon) \boldsymbol{R} & \mathbf{0} \\
\mathbf{0} & \mathbf{0} & \boldsymbol{G}_{v}^{-1}
\end{array}\right]
$$

ただし， $i=1,2$ (干同順).したがって，

$$
\lambda_{\min } \boldsymbol{N} \geq \lambda_{\min } \boldsymbol{N}_{1}, \lambda_{\max } \boldsymbol{N}_{2} \geq \lambda_{\max } \boldsymbol{N}
$$

となる.よって, 式（41）に代わって次式を得る. 
金岡克弥租弘明

Table 18 combinations with the eigen values of $\boldsymbol{N}_{i}(i=1,2)$

\begin{tabular}{|c|c|c|}
\hline Cases & $\lambda_{\min } \boldsymbol{N}_{1}$ & $\lambda_{\max } \boldsymbol{N}_{2}$ \\
\hline Case1 & $\lambda_{\min } \boldsymbol{G}_{v}^{-1}$ & $\lambda_{\max }\left(\boldsymbol{G}_{p}+\alpha \boldsymbol{G}_{v}+\frac{\alpha}{\epsilon} \boldsymbol{R}\right)$ \\
\hline Case2 & $\lambda_{\min } \boldsymbol{G}_{v}^{-1}$ & $\lambda_{\max }(1+\alpha \epsilon) \boldsymbol{R}$ \\
\hline Case3 & $\lambda_{\min }(1-\alpha \epsilon) \boldsymbol{R}$ & $\lambda \max \left(\boldsymbol{G}_{p}+\alpha \boldsymbol{G}_{v}+\frac{\alpha}{\epsilon} \boldsymbol{R}\right)$ \\
\hline Case4 & $\lambda_{\min }(1-\alpha \epsilon) \boldsymbol{R}$ & $\lambda_{\max }(1+\alpha \epsilon) \boldsymbol{R}$ \\
\hline Case5 & $\lambda_{\min }(1-\alpha \epsilon) \boldsymbol{R}$ & $\lambda_{\max } \boldsymbol{G}_{v}^{-1}$ \\
\hline Case6 & $\lambda_{\min }\left(\boldsymbol{G}_{p}+\alpha \boldsymbol{G}_{v}-\frac{\alpha}{\epsilon} \boldsymbol{R}\right)$ & $\lambda_{\max }\left(\boldsymbol{G}_{p}+\alpha \boldsymbol{G}_{v}+\frac{\alpha}{\epsilon} \boldsymbol{R}\right)$ \\
\hline Case7 & $\lambda_{\min }\left(\boldsymbol{G}_{p}+\alpha \boldsymbol{G}_{v}-\frac{\alpha}{\epsilon} \boldsymbol{R}\right)$ & $\lambda_{\max }(1+\alpha \epsilon) \boldsymbol{R}$ \\
\hline Case8 & $\lambda_{\min }\left(\boldsymbol{G}_{p}+\alpha \boldsymbol{G}_{v}-\frac{\alpha}{\epsilon} \boldsymbol{R}\right)$ & $\lambda_{\max } \boldsymbol{G}_{v}^{-1}$ \\
\hline
\end{tabular}

$$
\left\|\Delta x\left(t_{1}\right)\right\| \leq \sqrt{\frac{\lambda_{\max } \boldsymbol{N}}{\lambda_{\min } \boldsymbol{N}}} r_{1} \leq \sqrt{\frac{\lambda_{\max } \boldsymbol{N}_{2}}{\lambda_{\min } \boldsymbol{N}_{1}}} r_{1}
$$

そこで $\lambda_{\min } \boldsymbol{N}_{1}, \lambda_{\max } \boldsymbol{N}_{2}$ を考えると Table 1 に示すよう に八つの場合が存在する.

例えば, [Case2] のときは，

$$
\sqrt{\frac{\lambda_{\max } \boldsymbol{N}_{2}}{\lambda_{\min } \boldsymbol{N}_{1}}} r_{1}=\frac{4 \eta_{1} \sqrt{\lambda_{\max }(1+\alpha \epsilon) \boldsymbol{R}(\boldsymbol{q})}}{\sqrt{\rho}+\sqrt{\rho-16 \eta_{1} \eta_{3} / \rho}}
$$

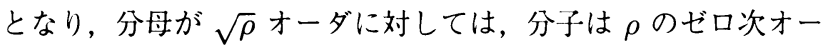
ダとなり, $\rho$ の増加とともに\| $\|x(t)\|$ の上限值は小さくなる. 他の [Case3] から [Case8] も同様に計算でき, 分母の $\rho$ のオー ダが異なるものの, $\rho$ の増加に従って, $\|\Delta x(t)\| の$ 上限值を小 さくすることができる。一方 [Case1] では,

$$
\begin{gathered}
\sqrt{\frac{\lambda \max \boldsymbol{N}_{2}}{\lambda_{\min } \boldsymbol{N}_{1}}} r_{1}=\frac{4 \eta_{1} \beta}{1+\sqrt{1-16 \eta_{1} \eta_{3} / \rho^{2}}}, \\
\beta=\sqrt{\lambda \max \left\{(a+\alpha) \boldsymbol{I}+\frac{\alpha}{\epsilon \rho} \boldsymbol{R}\right\}}
\end{gathered}
$$

となり, $\rho$ の増加に従って, $\|\Delta x(t)\|$ の上限値を小さくすること ができない。 それゆえに, 文献[7] と同様な考え方では, フィー ドバックゲインの増加によって, 軌道追従精度の改善は達成で きない.これはアクチュエータダイナミクスを考慮してリアプ ノフ関数に $\boldsymbol{G}_{v}^{-1}$ を含めたためである。そ枕えに，何らかの 別の方法で，軌道追従精度の向上を保証する必要がある。

そこで式（46）より適当な正数 $\theta$ によって以下の関倸に注意 する.

$$
\|\Delta x\| \leq \sqrt{\frac{\lambda \max \boldsymbol{N}_{2}}{\lambda_{\min } \boldsymbol{N}_{1}}} r_{1} \leq 2 \eta_{1} \beta \leq \theta
$$

この関数を用いて，式（26）を書き換える.

$$
\begin{aligned}
& \dot{V}(\Delta \boldsymbol{x}(t)) \\
& \quad \leq-\Delta \boldsymbol{x}^{T}(t)\left(\boldsymbol{M}-\theta \widetilde{\eta}_{3} \boldsymbol{I}\right) \Delta \boldsymbol{x}(t)+\Delta \boldsymbol{x}^{T}(t) \boldsymbol{\psi}
\end{aligned}
$$

両辺を積分して，

$$
\begin{aligned}
& \int_{0}^{t} \Delta \boldsymbol{x}^{T}(\tau) \boldsymbol{\psi}(\tau) d \tau \geq V(\Delta \boldsymbol{x}(t)) \\
& \quad+\int_{0}^{t} \Delta \boldsymbol{x}^{T}(\tau)\left(\boldsymbol{M}-\theta \widetilde{\eta}_{3} \boldsymbol{I}\right) \Delta \boldsymbol{x}(\tau) d \tau
\end{aligned}
$$

となる、さらに

$$
\begin{aligned}
& \frac{1}{2 \zeta} \int_{0}^{t} \Delta \boldsymbol{x}^{T}(\tau) \Delta \boldsymbol{x}(\tau) d \tau+\frac{\zeta}{2} \int_{0}^{t} \boldsymbol{\psi}(\tau)^{T} \boldsymbol{\psi}(\tau) d \tau \\
& \quad \geq \int_{0}^{t} \Delta \boldsymbol{x}^{T}(\tau) \boldsymbol{\psi}(\tau) d \tau
\end{aligned}
$$

を利用すれば，次式を得る。

$$
\begin{aligned}
& \frac{\zeta}{2} \int_{0}^{t} \boldsymbol{\psi}(\tau)^{T} \boldsymbol{\psi}(\tau) d \tau \geq V(\Delta \boldsymbol{x}(t)) \\
& \quad+\int_{0}^{t} \Delta \boldsymbol{x}^{T}(\tau)\left(\boldsymbol{M}-\theta \widetilde{\eta}_{3} \boldsymbol{I}-\frac{\boldsymbol{I}}{2 \zeta}\right) \Delta \boldsymbol{x}(\tau) d \tau
\end{aligned}
$$

さらに，式 $(34)$ と $V(\Delta \boldsymbol{x}(t)) \geq 0$ によって

$$
\begin{aligned}
& \frac{\zeta}{2} \int_{0}^{t} \boldsymbol{\psi}(\tau)^{T} \boldsymbol{\psi}(\tau) d \tau \geq V(\Delta \boldsymbol{x}(t)) \\
& \quad+\int_{0}^{t} \Delta \boldsymbol{x}^{T}(\tau)\left(\boldsymbol{M}-\theta \widetilde{\eta}_{3} \boldsymbol{I}-\frac{\boldsymbol{I}}{2 \zeta}\right) \Delta \boldsymbol{x}(\tau) d \tau \\
& \quad \geq \frac{1}{2} \int_{0}^{t} \Delta \boldsymbol{x}^{T}(\tau) \Delta \boldsymbol{x}(\tau) d \tau \\
& \quad+\int_{0}^{t} \Delta \boldsymbol{x}^{T}(\tau)\left(\boldsymbol{M}_{0}-\frac{1}{2} \boldsymbol{I}-\theta \widetilde{\eta}_{3} \boldsymbol{I}-\frac{\boldsymbol{I}}{2 \zeta}\right) \Delta \boldsymbol{x}(\tau) d \tau
\end{aligned}
$$

ここで, $\boldsymbol{M}_{0}$ の固有值は $\rho$ であるので,

$$
\frac{\rho}{2}>\frac{1}{2 \zeta}+\frac{1}{2}+\theta \widetilde{\eta}_{3}
$$

となれば，式（52）の右辺第 2 項は正となる。よって

$$
\zeta \int_{0}^{t} \boldsymbol{\psi}(\tau)^{T} \boldsymbol{\psi}(\tau) d \tau \geq \int_{0}^{t} \Delta \boldsymbol{x}(\tau)^{T} \Delta \boldsymbol{x}(\tau) d \tau
$$

式 $(53)$ より $\rho$ の増加とともに 有界であることを思い出せば，式（54）の $\boldsymbol{L}^{2}$ ノルムの意味で 軌道追従がゲインの増加によって実現できることが示せる.

最後に, $\|\Delta x(t)\|$ の上限値が, $r_{2}$ を超えないかを調べてお く必要がある。

$\eta_{2}=\frac{\rho}{2}$ とすると,

$$
r_{2}=\frac{\frac{\rho}{2}+\sqrt{\left(\frac{\rho}{2}\right)^{2}-4 \eta_{1} \eta_{3}}}{2 \eta_{3}}
$$

となるので，いずれの場合も $\rho$ を十分な大きさに設定すること によって，各上限の值を $r_{2}$ 以下とできる。

\section{4. 漸 近安定 性}

前章までで, $0 \leq t \leq T_{f}$ において, 目標軌道 $\boldsymbol{q}_{d}(t)$ に, フィー ドバックゲインを増大させることで任意の精度で追従可能であ ることが示せた。

次に, $t \geq T_{f}$ で, 運動が一定值 $\boldsymbol{q}_{d}\left(T_{f}\right)$ に収束するかについ て検討する。

$\dot{\boldsymbol{q}}_{d}=\ddot{\boldsymbol{q}}_{d}=\mathbf{0}$ の場合 $\boldsymbol{\psi}_{q}=\mathbf{0}$ となる. 仮に, $\boldsymbol{\psi}_{a}=\mathbf{0}$ とする. 重力項がもともと存在しない場合や, $\boldsymbol{H}(\boldsymbol{q}, \boldsymbol{q}, \boldsymbol{\tau})=$ 
$\boldsymbol{D}^{-1}(\boldsymbol{q}, \dot{\boldsymbol{q}}, \boldsymbol{\tau})$ かつ $\hat{\boldsymbol{A}}\left(\boldsymbol{q}_{d}, \dot{\boldsymbol{q}}_{d}\right)=\boldsymbol{A}\left(\boldsymbol{q}_{d}, \dot{\boldsymbol{q}}_{d}\right)$ の場合がこれにあ たる。 その際, 式 (35) は

$$
\dot{V}(\Delta \boldsymbol{x}) \leq-\left(\eta_{2}-\eta_{3}\|\Delta \boldsymbol{x}(t)\|\right)\|\Delta \boldsymbol{x}(t)\|^{2}
$$

となる. $t=T_{f}$ で安定な領域にあり，\| $\|\boldsymbol{x}(t)\|$ が有界である ことから $\eta_{2}=\frac{\rho}{2}>\eta_{3}\|\Delta \boldsymbol{x}(t)\|$ と $\rho$ を限定できる. よって, 漸 近安定性が保証できる。

\section{5. 空気圧システムの例}

ここでは，空気圧シリンダーシステムを対象に本論文で提案 した階層型フィードバックを用いて軌道追従制御のシミュレー ションを行う。その際, 制御入力を有効断面積とし, 今回はバル ブ，配管などによる遅れは対象モデルとして考慮しないで，お もに空気の非線形な圧縮性を考察する。

\section{1 機械システムのダイナミクス}

Fig. 1 で,ばねの自然長での位置を $q_{r}$ とした場合 $(q=0)$, 機械システムの運動方程式を以下に書く.

$$
\left(M_{L}+M_{P}\right) \ddot{q}+b \dot{q}+K q=\tau
$$

ただし， $\tau=S P, S$ : シリンダー断面積, $P$ : 圧力, $q(t):$ ピ ストンの変位, $M_{L}$ : 負荷の質量, $M_{P}$ : ピストンロッドの質量, $b:$ 粘性係数, $K:$ ばね係数. また, 以下ではピストンの目標軌 道を $q_{d}(t)$ とする.

\section{2 空気のダイナミクス}

アクチュエータ系のダイナミクスとなる空気圧の圧縮性は, 次 式で与えられる[1].

$$
\dot{\tau}+\kappa \frac{\dot{q}}{q} \tau=R T \frac{f}{q} u
$$

ただし， $u$ :バルブの有効断面積, $\kappa$ : 比熱比, $R$ : 気体定数, $T$ : 絶対温度, $f:$ バルブの非線形係数であり, バルブの有効断面 積を入力と見なすと非線形の一次遅れ系となる。ここで $f$ の詳 細は,

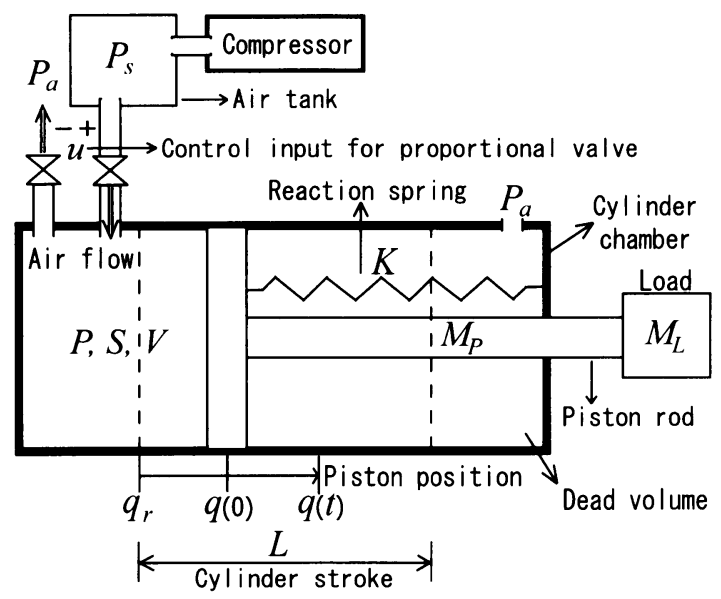

Fig. 1 Single-acting pneumatic cylinder system

$$
f=\left[\begin{array}{l}
c_{f 1} \text { if } \frac{P_{d n}}{P_{u p}} \leq P_{c r} \text { or, } \\
c_{f 2}\left(\frac{P_{d n}}{P_{u p}}\right)^{1 / \kappa} \sqrt{1-\left(\frac{P_{d n}}{P_{u p}}\right)^{(\kappa-1) / \kappa}}
\end{array}\right.
$$

ただし $P_{u p}$ は上流側圧力， $P_{d n}$ は下流側圧力を表し,

$$
\begin{gathered}
c_{f 1}=\sqrt{\frac{\kappa}{R}\left(\frac{2}{\kappa+1}\right)^{(\kappa+1) /(\kappa-1)}} ; c_{f 2}=\sqrt{\frac{2 \kappa}{R(\kappa-1)}} ; \\
P_{c r}=\left(\frac{2}{\kappa+1}\right)^{\kappa /(\kappa-1)}
\end{gathered}
$$

である。一方，

$$
V=S q \geq \epsilon>0
$$

と仮定する。ただし， $\epsilon$ は適当な正数とする。ここで,

$$
A(q, \dot{q})=\kappa \frac{\dot{q}}{q}, \quad D(q, \dot{q}, \tau)=R T \frac{f}{q}
$$

とおくことができ，

$$
A(q, \dot{q})-A\left(q_{d}, \dot{q}_{d}\right)=\kappa\left\{\frac{1}{q}\left(\dot{q}-\dot{q}_{d}\right)+\frac{\dot{q}_{d}}{q q_{d}}\left(q_{d}-q\right)\right\}
$$

と書ける.この場合, 式 (61) より $q \geq \frac{\epsilon}{S}>0$ となるので,

$$
\left\|A(q, \dot{q})-A\left(q_{d}, \dot{q}_{d}\right)\right\|=\xi_{1}\left\|\dot{q}-\dot{q}_{d}\right\|+\xi_{2}\left\|q-q_{d}\right\|
$$

となり，[条件 1] のリプシッツ条件を満足する．次に，仮定の式 (61) と $q_{d}(t)$ を適切に与えることによって [条件 2] の有界性も 満足される. 結局バルブの有効断面積を入力とする空気圧ダイ ナミクスは，本論文で想定する非線形ダイナミクスモデルとし て表現することができる， $\boldsymbol{D}(\boldsymbol{q}, \dot{\boldsymbol{q}}, \boldsymbol{\tau})$ については，f>0で $f$ は上流・下流側の圧力の関数となるが構造は既知であり, 有界 でもある，したがって，[条件 3] を满たす $\boldsymbol{H}(\boldsymbol{q}, \boldsymbol{q}, \boldsymbol{\tau})$ を容易に 設計できる. 以下では, $H(q, q, \tau)=1$ とする.

\section{3 制御則}

まず，以下に仮想的な目標圧力を位置および速度偏差を用い て構成する。

$$
\tau_{d}=S P_{d}=-G_{p} \Delta q-G_{v} \Delta \dot{q}+K q_{d}(t)
$$

ここで, $\Delta q(t)=q(t)-q_{d}(t)$ である. 次に，制御入力を上記 の仮想目標圧力と圧力測定値を用いて生成する.

$$
u=K_{p}\left(\tau_{d}-\tau\right)=K_{p} S\left(P_{d}-P\right)
$$

ただし, 式 (66) において, 運動終了時 $(\dot{q}=0)$ は $A\left(q_{d}, \dot{q}_{d}\right)=0$ になるため, 式 (13) の $\boldsymbol{\psi}_{a}$ の $\hat{A}\left(q_{d}, q_{d}\right)$ 項はいらない. 
Table 2 Physical parameters of the cylinder model

\begin{tabular}{|l|l|}
\hline Mass $\left(M_{L}+M_{P}\right)$ & $M=15[\mathrm{~kg}]$ \\
\hline Cylinder cross sectional area & $S=12.56\left[\mathrm{~cm}^{2}\right]$ \\
\hline Temperature & $T=293[\mathrm{~K}]$ \\
\hline Viscous friction coefficient & $B=0.033[\mathrm{kgf} \mathrm{s} / \mathrm{cm}]$ \\
\hline Spring constant & $K=2.54[\mathrm{~N} / \mathrm{cm}]$ \\
\hline Stroke & $L=30[\mathrm{~cm}]$ \\
\hline Initial position & $q(0)=10[\mathrm{~cm}]$ \\
\hline Initial gauge pressure & $P_{0}=202.6[\mathrm{kPa}]$ \\
\hline Supply gauge pressure & $P_{s}=607.8[\mathrm{kPa}]$ \\
\hline Atmospheric gauge pressure & $P_{a}=0[\mathrm{kPa}]$ \\
\hline Control input saturation & $\left|u_{\max }\right|=2.5\left[\mathrm{~mm}^{2}\right]$ \\
\hline
\end{tabular}

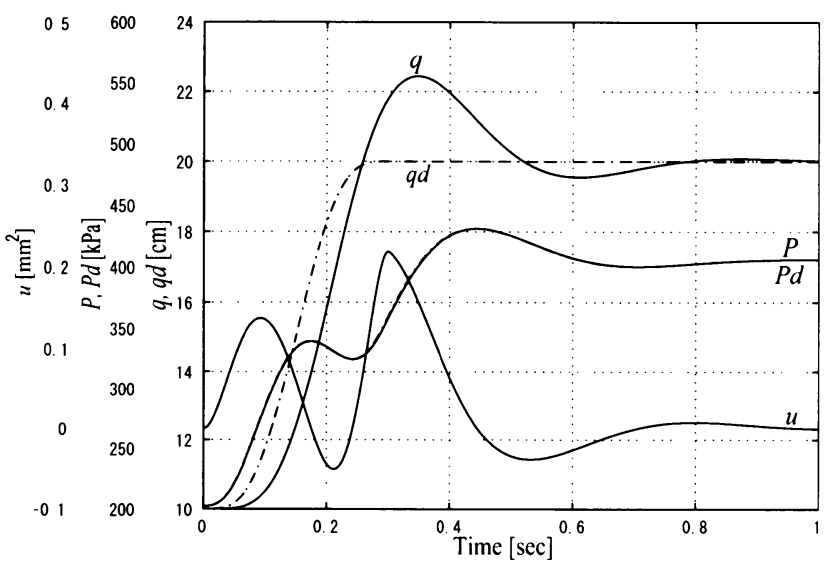

Fig. 2 Trajectory tracking result of $K_{p}=150, G_{p}=150$, $G_{v}=150$ by cascaded feedback control with input limitation

\section{4 シミュレーション}

Fig. 1 のシステムにおいて, 本稿で提案する制御則（式 (65), (66)）を用いて，以下の目標軌道,

$$
\begin{aligned}
q_{d}(t)= & -9144.9 t^{7}+9602.2 t^{6} \\
& -3456.8 t^{5}+432.1 t^{4}+0.1[\mathrm{~m}]
\end{aligned}
$$

にての軌道追従制御シミュレーション結果を以下に示す。ここ では, 滑らかな入力の生成のため, 目標軌道を $\mathrm{C}^{3}$ 級にしてい る.ただし，ゲインの単位は $G_{p}[\mathrm{kN} / \mathrm{m}], G_{v}[\mathrm{kN} \mathrm{s} / \mathrm{m}], K_{p}$ $\left[\mathrm{mm}^{2} / \mathrm{MN}\right]$ である. シミュレーションのパラメータは Table 2 である.また, 式 $(60)$ において比熱比 $\kappa=1.4$, 理想気体定 数 $R=0.2872$ にしている. 式（58）で表現される空気のダイ ナミクスは, 式 $(61)$ の仮定をおけば，本論文で取り扱う理論 が適用できる。ただし, 実際に利用されるシステムでは, 入力 量が制限される場合やバルブのダイナミクスが無視できない場 合もある。そこで，本論文では入力量に制限がある場合，すな わちバルブの開口面積に実際の制限がある場合を想定に, Table 2 に示されるパラメータ值でシミュレーションを行った. シミュ レーションに用いたパラメータ值と目標軌道は高慣性, 高速で あるので，一般的に制御が難しい対象として設定している。

\section{5 シミュレーション結果}

まず入力量に制限がない場合, ゲインの上昇と伴って軌道追 従制御性能が上げられることが確認できた。また, 式 (29) を計 算した結果では, 式（29）を正にしていて，不安定に陥ることな

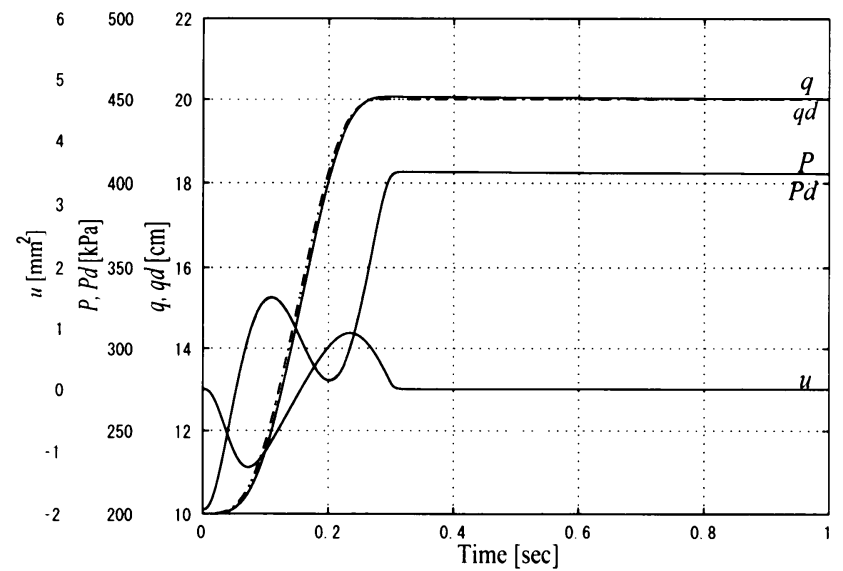

Fig. 3 Trajectory tracking result of $K_{p}=2300, G_{p}=2300$, $G_{v}=2300$ by cascaded feedback control with input limitation

く軌道追従性能が改善できていることを確認した. ゲインの初 期設定においては，ダイナミクスパラメータ值が概略知られて いれば，ゲインチューニングとして，まず一段目の機械ダイナ ミクスの $G_{p}, G_{v}$ を 2 次遅れ系として設計できる. 次に, 二段 目のゲイン $K_{p}$ を式 $(29)$ が最低限, 正になるように設定した. 次に入力量に制限ありの場合において，ゲインの上昇とともに 軌道追従性能が改善できる傾向が確認できた（Fig. 2, Fig. 3). また，一般的な PD 制御則，

$$
u=-G_{p} \Delta q-G_{v} \Delta \dot{q}+K q_{d}(t)
$$

として，ゲインチューニングを試みたが，Fig. 3 に見られる立 ち上がりの精度を得るところまでゲインを上げるとシステムが 不安定となり適切なゲインが得られなかった。

\section{6.おわりに}

ロボット運動制御において，アクチュエータ系ダイナミクス が無視できない場合の軌道追従制御と目標位置制御に対して, 階層的のフィードバック制御の有効性を示した。重力補償につ いては軌道追従の目的のみであれば必ずしも正確なパラメー夕 值を用いる必要はない。一方，位置制御では， $t \geq T_{f}$ におい て $\boldsymbol{\psi}=\mathbf{0}$ とした．実際では, $\boldsymbol{A}(\boldsymbol{q}, \dot{\boldsymbol{q}}), \boldsymbol{D}(\boldsymbol{q}, \dot{\boldsymbol{q}}, \boldsymbol{\tau})$ の各要素 の推定が正確である保証はない。これらの場合はPID 制御など を利用すべきで，別の機会に報告する．アクチュエータ系への フィードバックゲイン $\boldsymbol{K}_{p}$ は $\rho^{2}$ オーダとなり, $\boldsymbol{G}_{p}, \boldsymbol{G}_{v}$ の $\rho$ オーダに比べて，極端に大きな値を設定することを要求する場 合が起こり得る．実際に設定すべき值と理想的に保証すべき值 の差が大きく存在するかなどの検討は今後の課題としたい.

\section{参 考 文 献}

[1] S. Kawamura, K. Miyata, H. Hanafusa and K. Ishida: "A Hierarchical Feedback Control Scheme for Pneumatic Drive Systems," SICE, vol.26, no.2, pp.204-210, 1990

[2] M. Moallem and J. Lu: "Application of Shape Memory Alloy Actuators for Flexture Control," IEEE/ASME Transaction on Mechatronics, vol.10, no.5, pp.495-501. 2005.

[3] T.I. Fossen: Guidance and Control of Ocean Vehicles. JOHN 
WILEY \& SONS, 2005.

[4] R. Ortega, A. Loria, P.J. Nicklasson and H. Sira-Ramirez: Passivity-based Control of Euler-Lagrange Systems. Springer, 1998.

[5] S. Arimoto: Control Theory of Non-linear Mechanical Systems. OXFORD Univ. Press, 1996.

[6] S. Kawamura, F. Miyazaki and S. Arimoto: "Is a local PD feedback control law effective for trajectory tracking of robot motion," Proc. of IEEE Conf. on Robotics and Automation, pp.1335-1340, 1988.

[7] D.M. Dawson, Z. Qu, F.L. Lewis and J.F. Dorsey: "Robust control for the tracking of robot motion," INT. J. CONTROL, vol.52, no.3, pp.581-895, 1990.

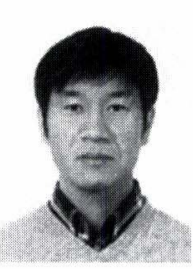

全 珍雨 (Jinwoo Jun)

1973 年 3 月 9 日生. 1999 年韓国のハンヤング大学 機械工学科卒業. 同年同国の工場自動化関連会社で 技術営業職として 2 年間勤務. 2004 年立命館大学 大学院理工学研究科情報システム学専攻修士課程修 了. 現在同大学大学院理工学研究科総合理工学専攻 博士後期課程在学中. (日本ロボット学会学生会員)

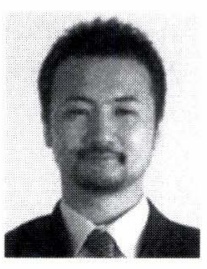

\section{金岡克弥 (Katsuya Kanaoka)}

1971 年 7 月 31 日生. 2002 年京都大学大学院工学 研究科博士後期課程研究指導認定退学. 2003 年立 命館大学理工学部ロボティクス学科講師, 現在に至 る. 水中ロボット, パワー増幅ロボットの研究に従 事. 博士 (工学)。システム制御情報学会, 日本機 械学会の会員。

(日本ロボット学会正会員)

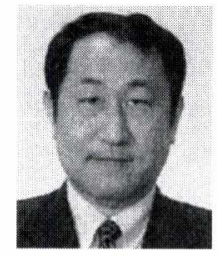

川村貞夫（Sadao Kawamura）

1956 年 7 月 10 日生. 1981 年大阪大学基礎工学部 生物工学科卒業. 1986 年同大学大学院博上課程修 了. 工学博士. 同年大阪大学基礎工学部助手. 1987 年立命館大学理工学部機械工学科助教授. 1995 年 同大学教授. 学習制御, ロボットの運動制御, 空気 压駆動システム, ワイヤ駆動システム, 人間運動特 性の計測・解析などの研究に従事. IEEE，システム制御情報学会，日 本機械学会, 計測自動学会などの会員。(日本ロボット学会正会員)

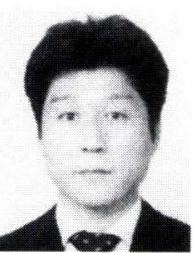

櫟弘明 (Hiroaki Ichii)

1966 年 7 月 4 日生. 1991 年立命館大学理工学部情 報工学科卒業. 1993 年同大学大学院理工学研究科 情報厂学専攻修了。同年財団法人京都高度技術研究 所研究員. 1997 年国立奈良高等専門学校助手. 現 在同学校助教授. 学習制御などの研究に従事. 計測 自動制御学会の会員。(日本ロボット学会正会員) 